\title{
Study on an information system of vegetable safety production and management
}

\author{
ZHANG Yihua ${ }^{1, a}$, LI Dongming ${ }^{2}$,WANG Yu ${ }^{3}$,LIU Jun ${ }^{3}$, Gao Liang ${ }^{4}$ \\ ${ }^{1}$ JIBEI Baoding Electric Power VOC. \&TECH. College, Baoding, 071051, China \\ ${ }^{2}$ College of Mechanical and Electrical Engineering, Hebei Agricultural University, Baoding, 071001, \\ China \\ ${ }^{3}$ SIKE Agricultural Technology Co., Ltd, Langfang, 065000, China \\ ${ }^{4}$ Administration Department of science and Technology, Hebei Agricultural University, Baoding \\ 071001, China \\ aemail:1147439296@qq.com
}

Keywords: vegetable, safety production, information system, 2D code

\begin{abstract}
Establishing complete quality supervision of vegetable production is an important measure and method to improve the quality of vegetables and ensure food safety. In this paper, an information system of vegetable safety production management has been designed, which has provided comprehensive information service from vegetable production to consumption. Linux + Apache + MySQL + PHP method was adopted in the system. Linux was an operating system, Apache was a server, MySQL was the database, and PHP was language used to connect each other. There was B/S 3-layer architecture including performance layer, business logic layer and data layer. QR 2D code with high information capacity was used to manage and trace back to vegetable production information. The encryption and error correction method of QR codes was studied, and a kind of hybrid algorithm based on AES and ECC two encryption algorithms was proposed. The system has provided technical support for informatization and network management in the process of vegetable safety production, strengthened the government supervision, standardized the production management of enterprises and improved the consumer's right to know.
\end{abstract}

\section{Introduction}

China is a big country of vegetable production, and vegetable industry plays an important role in agricultural production. The quality of vegetables is not only related to people's health and life safety, but also has a significant impact on agricultural development and social stability. The mode of vegetable production must be transformed from high-input for high-yield to high-nutrition, high-quality, high-efficiency and non-pollution. Furthermore, it is necessary of production technology standard without pollution of quality and nutrition to support and promote the healthy development of vegetable industry. To realize fine quality production of vegetables and safety consumption of the consumers, it is also necessary to establish perfect management information system of vegetable safety production. The system can provide transparence production information and realize the whole process quality monitoring from the fields to the dining-table.

In the paper, an information system of vegetable safety production management was built with agricultural information intelligent service technology, with vegetable information being a starting point, the collection of information being the basis, information processing being a crucial part, and accurate service being the purpose. The system used 2D code technology to store the information of the various links of vegetable production and management, which realize the efficient storage, convenient spread and rapid identification of information. Consumers could accurately scan the relevant information through the mobile phone, which provided reference for eating with safety. Quality superintending department could supervise and manage safety production of vegetables to improve the quality by reading of analyzing information. The system could collect, arrange, store and analyze to realize the network of information management and the intelligent information inquiry. 
The system provided ancillary decision-making services for researchers, technical support for producers, and consulting service of safety food for customers, so the system possesses a higher applied value.

\section{Application of $2 \mathrm{D}$ code technology}

2D code could express all kinds of information such as text, images and audio. The information of vegetable in all stages was generated and transformed into 2D code, which was simple to form, convenient to use and didn't need hardware equipment investment. 2D code, which has abilities of high information capacity, high speed full range reading and error correction, was used as the carrier to realize the online and offline synchronization monitoring of the vegetable safety production management information system to the vegetable supply chain composed of producers, processors, sellers. In the meantime, consumers can clearly trace back to vegetables detailed information by 2D code and platform.

Quick Response (QR) code, a kind of 2D code, was invented by the Japanese DENSO WAVE company in 1994, and it was widely used at present. Compared with other 2D code, QR code has a large amount of advantages such as high data density and small occupied space; quick reading speed; 360-degree high speed reading; adaptation in the limited printing area and slender space printing needs.

\subsection{Encoding process of $Q R$ code}

Data analysis: at first, the input data was analyzed; and the type of encoded character was determined and transformed into symbol character; finally error correction level was selected. According to the length of the data code and error correcting code, the appropriate QR code symbol version was selected.

Data encoding: in accordance with the definition rules of model used, the data character was converted to a bit stream. In the process of mode conversion, mode indicator was added at the beginning of new mode, and the terminator was added to after the data sequence. When the data character was converted into a bit stream, there were 8 bits in every code, and character string formed a sequence of data.

Golay encoding: according to the need the above code sequence was divided into blocks; and according to the error correction level and block code, error correction code was generated. The error correction code was added behind data code sequence, which became a new sequence.

Final figure construction: in the condition of specification, the above sequence was put into different blocks. According to the regulations, the data was divided into blocks, and each was calculated to get the corresponding error correcting code block, which made up a sequence to was added behind the original data sequence.

Permutation code: the separator, the positioning graphics, the corrected graphics, the detection figure and the codeword module were put into the matrix.

Masking: in order to enhance the reliability of the recognition, masking mode was used for a balanced arrangement of bright and dark module, the ratio of which closed to 1:1. This arrangement minimized the difficult of decoding, and at the same time, and position detect image bit stream should be avoided in the data area.

Format and version information: format and version information was put into the appropriate area. There was version information in version 7-40, and version 0 has no information. Version information had 18 bits, six bits were data bits and the rest 12 bits were the error correction.

\subsection{QR code-encryption}

The information at all stages of the product contained in the QR code should not be arbitrarily changed, so it was necessary to add encryption algorithm to QR code in the process of encoding. According to the research of various encryption algorithms, this paper used hybrid algorithm based on AES and ECC two encryption algorithms to encrypt 2D code. Advanced Encryption Standard

(AES ) was fast and safe. Elliptic Curves Cryptography (ECC) was the elliptic curve cryptography encoding, producing 2 keys to encryption and decryption. AES was relatively fast, which was suitable 
for the characteristics of long information chain; at the same time, ECC could be used to manage the key, so the hybrid encryption algorithm was proposed, which improved the security of data encryption as well as improved the rate of data encryption. Mixed encryption process diagram shown in figure 1.

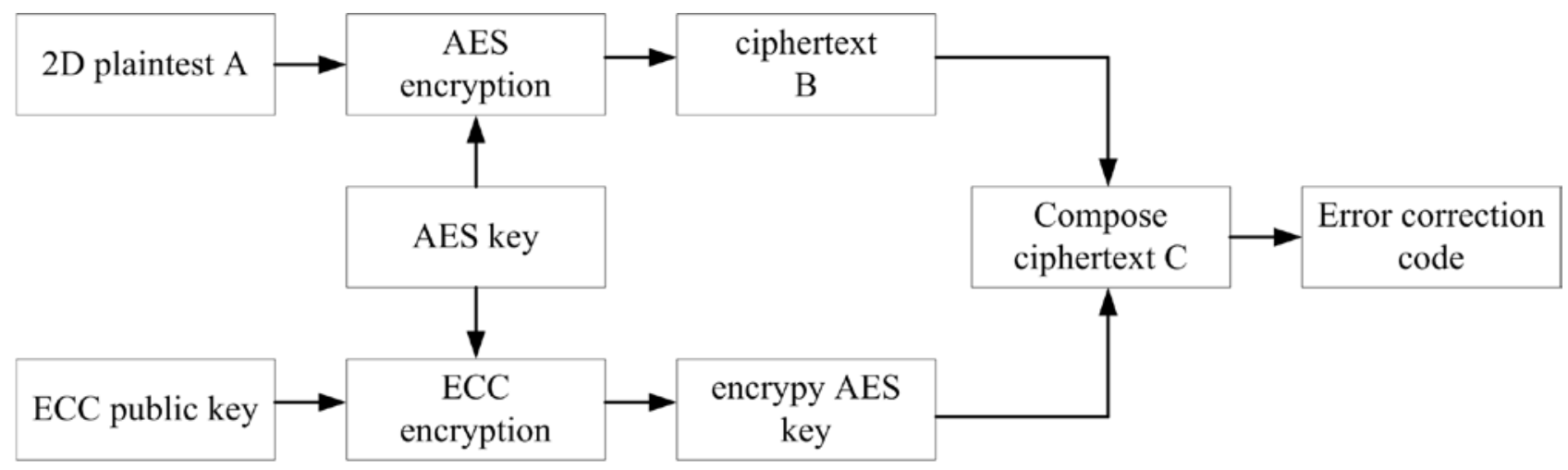

Fig.1 AES- ECC Mixed encryption process diagram

\subsection{Error correction of QR code}

QR code could be damaged and stained in the production and circulation, which had a negative impact on the code identification. Therefore, it was important to choose a good error correction algorithm. The paper adopted Reed Solomon (RS ) error control codes, the highest error correcting capability of which was over $30 \%$.

Galois Field (GF) with q as prime was denoted by $G F(q)$.If a conformed to $a^{q-1}=1$, a was primitive element of $G F(q)$, and cyclic group was

$$
G(a): a^{0}, a^{1}, a^{2}, \cdots a^{q-1}
$$

If $\mathrm{k}$ errors were corrected in RS code, polynomial generated was

$$
g(x)=(x-a)\left(x-a^{2}\right)\left(x-a^{3}\right) \cdots\left(x-a^{2 k}\right)
$$

The polynomial of the information code was

$$
d(x)=d_{0}+d_{1} x^{1}+d_{2} x^{2}+d_{3} x^{3}+\cdots+d_{n-1} x^{n-1}
$$

The polynomial after RS encoding was

$$
h(x)=x^{2 k} d(x)+x^{2 k} d(x) \bmod g(x)
$$

In the polynomial, $x^{2 k} d(x)$ was the original code, and 3was the error correction code.

\section{System architecture design}

The information system of Vegetable safety production management as a whole used Linux + Apache + MySQL + PHP (LAMP). It was designed B/S three-layer architecture, which included information platform performance layer, business logic layer and data layer. It was shown in figure 2 . As combination of LAMP had increasing applications, the system had more and more compatibility and became a powerful WEB application platform. Linux was an operating system, Apache was a server, MySQL was the database and PHP was their language connecting each other. The remarkable 
feature of LAMP was open source, and the system architecture was widely used. Distributing, cloud computing, big data and to IOE was today's big trend, and the open source architecture was sought after by domestic and foreign Internet companies. Vegetable safety production and management information system used LAMP to realize complete system function and higher comprehensive performance

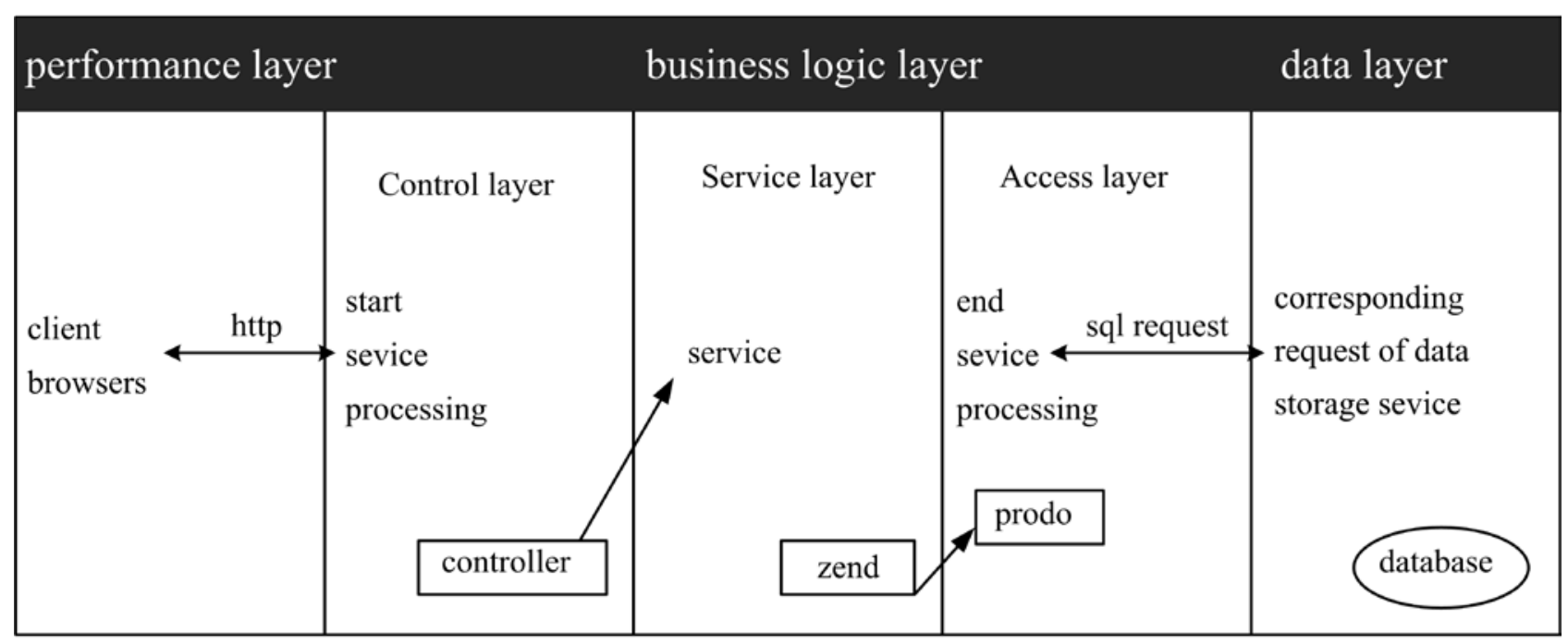

Fig.2 System architecture

\subsection{Performance layer}

Performance layer displayed and received data and also provided operable, friendly and visible functional interface for users. After the user input request, the results would be displayed to the user through the clients. The management system could be provided information for the government and enterprise internal users through the user interface to realize the government supervision and enterprises' management, and inquiry service for consumers based on the production of vegetable information.

\subsection{Business logic layer}

The business logic layer processed data requests sent out from the performance layer, communicated with the performance layer through the HTTP/HTTPS protocol, and was connected with the database. Business logic layer was divided into control layer, service layer, access layer. This layer was the core of the whole system, the position of the business object and the rules of application in the business object. Its task was to accept the request of the performance layer, according to the business rule processing request, to interact with the database and to pass results back to the performance layer.

\subsection{Data layer}

Data layer was mainly responsible for data management that was data classification, organization, encoding, storage, retrieval and maintenance, and it was the core of the data layer. Interacted with the performance layer and the logic layer; that was, data treated by the database server was sent back to the business logic layer, in which data was processed; the final results was sent to the performance layer to display to the user.

\section{Design of system function}

\subsection{Design of the functional unit}

The system could realize the unified management of vegetable production information, and realized the inquiry function through $2 \mathrm{D}$ code. The system consisted of several functional units: the vegetable producers' management files subunit, the government supervision subunit, traceability 
inquiry subunit, and production technology standardization subunit. Structure diagram of system function was shown in figure 3.

Vegetable producers' management files subunit mainly collected, collated and stored all kinds of information including farmers' information, land information, agricultural materials' information, processing information and so on in the vegetable growth and processing. Farmers and processors needed to input the information. The subunit also provided vegetable information inquiry function for consumers.

Government supervision subunit mainly collected stored, queried vegetables' inspection and quarantine results and information. Government regulators needed to log in as a verification, audited and assessed upload quality inspection information. Ordinary users could also query the relevant results in this subunit.

Traceability inquiry subunit provided vegetable information inquiry function for users. 2D code label affixed on the vegetable was generated in this sub unit. After users verified the identity in the login module, they could log in label generation module to generate a 2D code label.

Production technology standardization subunit provided users with the relevant standards, knowledge and technology. The unit mainly included the production technical standards, planting technology, knowledge at home and abroad, pesticide indicators, input information etc.

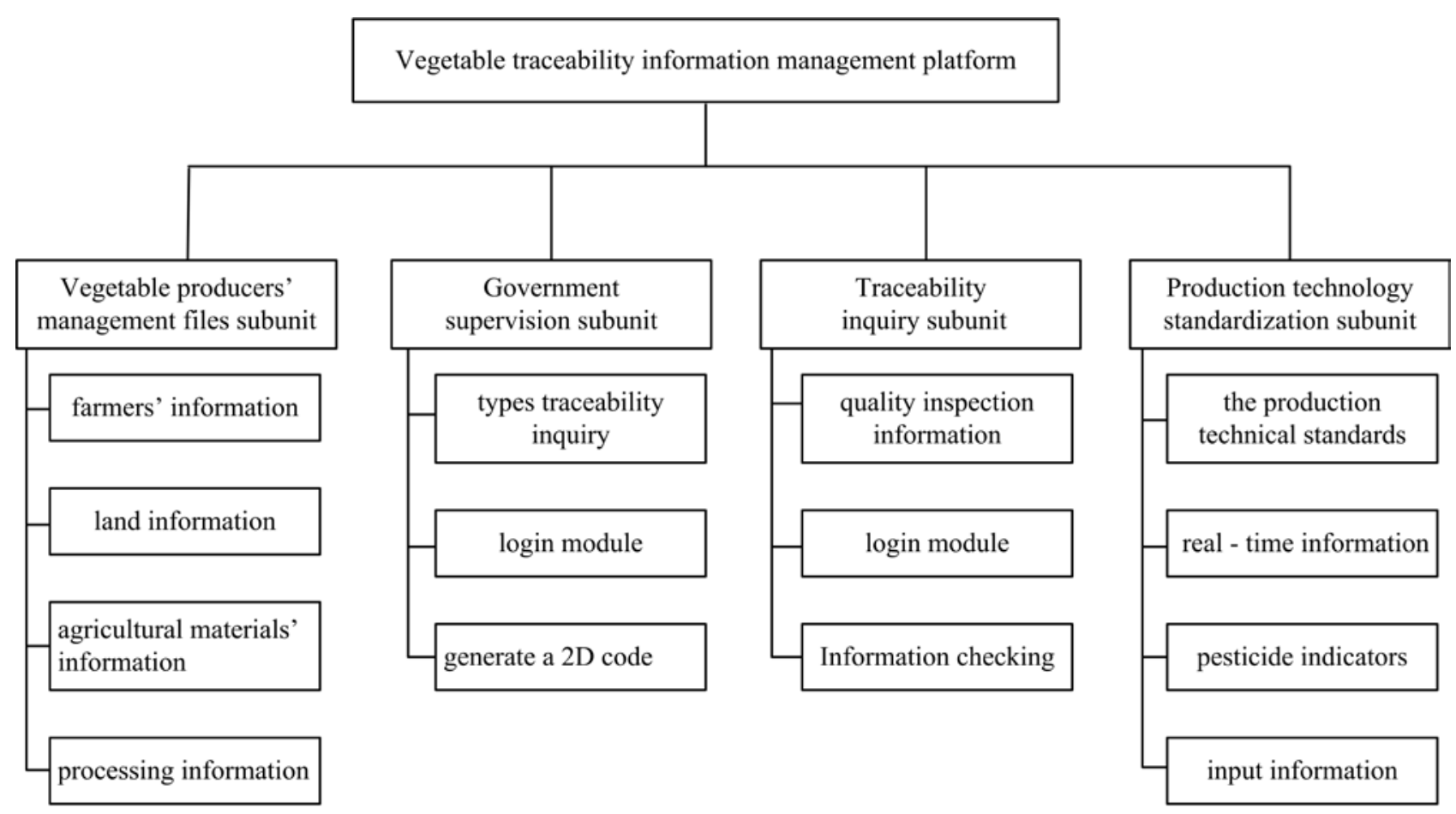

Fig.3 Structure diagram of system function

\subsection{Database design}

The efficient management of various information data was one of the core functions of the system. In the meantime, the reliability, security, and rapidity of data storage was put forward higher requirements. There was a great deal of safe production management information to store because of wide variety of vegetables. According to actual needs, the system used MySQL database. MySQL was management system of a small relational database, and its source code was open. Because of its low cost, small size, open source, high speed and other characteristics, MySQL was widely used in the Internet website construction. It was a multi-thread, fast, support-multi-users and powerful database server, and could be embedded into a large configuration software.

The database design was carried on according to the classification principle, which meant the same data table was put into the same level database, and it was convenient for data management and invocation. There were the database of vegetable production, the database of processing, the database 
of quality, the database of sales and the database of the information source in the system. These databases were primary databases, the lower level of the database was secondary database.

\section{Conclusions}

A management information system of vegetable safety production was designed in the paper, providing deep and round information services including the whole process of vegetable production, processing, circulation, sales and consumption. The system adopted B/S three-layer structure to realize the interaction, using PHP language to develop, selecting Apache as the server and MySQL as database, and using HTTP to communicate between the user and the server. QR 2D code with high information capacity was used to realize the traceability management for the information of vegetable safety production. The system has provided technical support for informatization and network management in the process of vegetable safety production, strengthened the government supervision, standardized the production management of enterprises and improved the consumer's right to know.

\section{References}

[1]LU Lei and ZHANG Feng. Design and implementation of vegetable traceability system based on internet of things. in: Electronic Design Engineering, Vol.19 No.7(Apr.2011), p19

[2]WANG Dong, ZHU Xangxian and Qian Xin. Meat and Vegetables Circulation Traceability System Based on Internet of Things. in:Hubei Agricultural Sciences, Vol.52 No.24(Dec.2013), p6167

[3]YANG Yong and ZHAO Xiuping. Organic Vegetables Traceability Management System Based on RFID Technology. in:CHINA PARINTING AND PACEAGING STUDY, Vol.06 No.4(2014),p60

[4]HE Yujie and LIANG Qi. Database Theory and Application. China Machine Press, 2011, p12-45

[5]YANG Xinting, QIAN Jianping, SUN Chuanhe, et al. Design and Application of Safe Production and Quality Traceability System for Vegetable. in:Transactions of the CASE, 2008, 24(3), p162-166

[6]SHEN Guanglei, ZAN Linsen, DUAN Junbiao, et al.. Implementation of Beef Quality and Safety Traceability System via Internet. in: Transactions of the CSAE, 2007,23(7), p170-173

[7]GE Wenjie, ZhAO Chunjiang. State-of-the-art and developing strategies of agricultural internet of things. Transaction of the Chinese Society for Agricultural Machinery, 2014, 45(7), p222-230

[8]Qin Huaibin, LI Daoliang, GUO Li. Recent advances in development and key technologies of internet of things in agriculture. in:Journal of Agricultural Mechanization Research, 2014(4), p246-248

[9]WANG Wei, Safety of Aquatic Products Production Information Transmission Key Technology Research. in:Taian Shandong Agricultural University, 2014

[10]DONG Fangmin, WANG Jihua, REN Dong. The technology and application of agricultural internet. Beijing , CHINA AGRICULTURE PRESS, 2012:132

[11]Kumar S, Iyengar S S, Lochan R, et al. Application of sensor networks for monitoring of rice plants: A case study. in: The $4^{\text {th }}$ International Symposium on IRADSN, 2009 